\title{
Damage-free Ablation Process for Back-contacted Silicon Heterojunction Solar Cells
}

\author{
Aryak Singh, Bugra Turan, Stefan Haas, Andreas Lambertz, Kaining Ding and Uwe Rau \\ IEK5 - Photovoltaik, Forschungszentrum Jülich GmbH, Jülich, 52425, Germany \\ Email: a.singh@fz-juelich.de
}

\begin{abstract}
This work explores the feasibility of ultra-short femto-second laser pulses in the green and infrared wavelength range $(515 \mathrm{~nm}, 1030 \mathrm{~nm})$, and nano-second pulses $(355 \mathrm{~nm})$ in the ultra-violet wavelength range to process back-contacted silicon heterojunction solar cells. Two hardmasks - a single silicon dioxide $\left(\mathrm{SiO}_{2}\right)$ layer and a combination of silicon dioxide and a hydrogenated amorphous silicon (a-Si:H) layer - were compared for a damage-free structuring process. We characterized the quality of ablation and its impact on the device properties by utilizing light and atomic force microscopy, Raman spectroscopy, and photoluminescence imaging. Light and atomic force microscopies were used to identify surface modification, ablation and damage regions. Threshold fluence for these zones was evaluated for both the hardmasks. From Raman spectroscopy no modification in the crystalline structure of the silicon substrate was observed with $\mathrm{SiO}_{2}+\mathrm{a}-\mathrm{Si}: \mathrm{H}$ mask after laser irradiation with single pulses. Square regions of $10 \times 10 \mathrm{~mm}^{2}$ were structured in the hard mask by separate and partially overlapping pulses. Using photoluminescence lifetime imaging, we successfully obtained damage-free processing windows of laser fluences and optimal spot overlap distances between adjacent pulses for the back-side emitter and base contact patterning of back-contacted silicon heterojunction solar cells.
\end{abstract}

DOI: 10.2961/jlmn.2018.03.0029

Keywords: a-Si/c-Si heterojunction, laser ablation, interdigitated back-contacts, laser patterning

\section{Introduction}

The current world record efficiency of $26.7 \%$ [1] for mono-crystalline silicon solar cells is based on the Interdigitated Back-contacted Silicon Heterojunction (IBC-SHJ) concept. However the industrialization of this device concept still remains a challenge. It requires the patterning of base and emitter regions of opposite polarity $(\sim 25 \mathrm{~nm} n-$ doped and p-doped amorphous silicon thin films) into interdigitated fingers on the back side of the solar cell without causing damage to the surface of the silicon wafer. Photolithography is a common method to fabricate high efficiency IBC-SHJ solar cells [2]. However, photolithography has a large number of processing steps, which increase the overall cost of fabrication. Laser ablation can be an attractive tool for the low-cost mass production of IBC-SHJ solar cells because it enables a very precise and selective removal of thin films, and can greatly reduce the number of fabrication steps as compared to photolithography.

The surface of a crystalline silicon (c-Si) wafer has non-saturated (dangling) bonds which induce a large number of defects states within the bandgap. These surface states act as recombination centers during a solar cell's operation. To avoid excessive efficiency losses, these surface states need to be well passivated. In silicon heterojunction solar cells, passivation is achieved by means of a few nanometers thin hydrogenated amorphous silicon (a-Si:H) film [3]. An undamaged interface between crystalline silicon (c-Si) wafer and a-Si:H film is crucial to prevent the photo-generated electrons and holes from recombining. Therefore, laser ablation is preferred away from this crucial a-Si:H/c-Si interface. The standard approach for laser pro- cessed IBC-SHJ solar cells is to locally ablate a laser light absorbing hard-mask and wet-chemically etch the underlying amorphous silicon films to pattern the emitter and base regions. This approach has been studied by previous groups using pulse durations in nano-second $(\lambda=532 \mathrm{~nm}, 355 \mathrm{~nm})$ [4] and pico-second $(\lambda=355 \mathrm{~nm})$ [5], [6] regimes. In these reports damage to the a-Si:H/c-Si interface has been reported, especially in the overlapping zone between adjacent laser pulses. To minimize the damage in this overlapping zone, a stacking of multiple hardmasks [5] or the use of a distributed Bragg reflector layer [6] has been proposed which, in turn, increase the complexity of processing.

The aim of this work was to achieve a damage free ablation without a complex processing sequence. We compared femto-second $(\lambda=515 \mathrm{~nm}, 1030 \mathrm{~nm})$ and nano-second laser pulses $(\lambda=355 \mathrm{~nm})$, as well as two different hardmask structures for the patterning of base and emitter contact regions in IBC-SHJ solar cells.

Ultra-short femto-second laser pulses have very short energy deposition times and are known to produce only minor heat affected zones [7]. Nevertheless, commercially available femto-second systems are still quite expensive and this might limit their applicability for a cost-effective and industrial IBC-SHJ fabrication sequence. Nano-second lasers on the other hand have larger heat affected zones but they cost a fraction of the cost of femto-second laser systems [8]. Ultra-violet nano-second lasers have a short optical penetration depth in silicon and they may be a good trade-off between the cost of the system and the damage to the passivation quality. 
a) Deposition

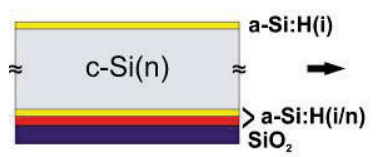

$\mathrm{SiO}_{2}$ hardmask

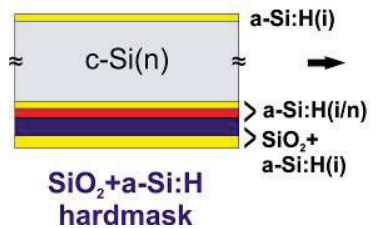

b) Laser scribe

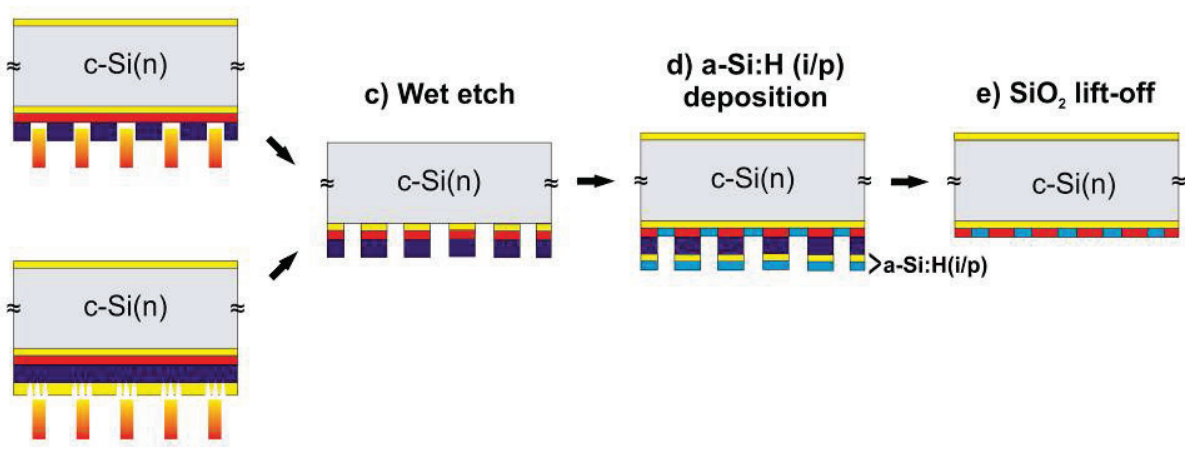

Fig.1 Processing sequence of $\mathrm{SiO}_{2}$ and $\mathrm{SiO}_{2}+\mathrm{a}-\mathrm{Si}: \mathrm{H}$ hard-mask for patterning emitter and base regions. a) deposition of amorphous films and hard masks b) laser ablation of hardmasks c) chemical treatment to etch base region d) a-Si:H (i+p) emitter deposition and e) lift-off of $\mathrm{SiO}_{2}$ film to separate a-Si(i+n) and a-Si:H(i+p) regions.

The hardmasks that we compare are a highly transparent but simple to fabricate silicon dioxide layer, and a combination of silicon dioxide and an absorbing amorphous silicon layer (Fig.1). Even though $\mathrm{SiO}_{2}\left(\mathrm{E}_{\mathrm{g}}=9.3 \mathrm{eV}\right)$ is transparent to light in the visible region, ultra-short pulses might lead to non-linear absorption in the dielectric film. Previous groups have studied the ablation behavior of $\mathrm{SiO}_{2}$ films on silicon and have reported negligible damage to the wafer after ablation[9],[10]. In the second hardmask, an absorbing amorphous silicon layer is introduced to contain the laser ablation and prevent any damage to the passivation quality at the a-Si:H/c-Si interface.

The approach that we adopted was to first understand the microscopic properties, such as visual damage, structural damage and the ablation characteristics of single laser pulses. We used Atomic Force Microscope (AFM) and Light Microscope (LM) to analyze the crater morphology of single-shot ablation craters and to calculate the threshold fluences. Micro Raman spectroscopy was utilized to characterize the structural changes and induced stress in the cSi substrate after ablation of the hardmask.

The microscopic analysis was then followed by a study of the impact of patterning on the passivation quality on a macroscopic scale. For our experiments we followed the laser ablation based LEAP processing sequence for IBCSHJ solar cell, as proposed by Ring et al [5]. Square areas were processed in the hardmasks with separate and overlapping pulses using a range of fluence values. The degradation in the minority carrier lifetimes under these laserprocessed regions was studied using photoluminescence (PL) imaging technique.

We found out that single laser pulses with both nanoand femto-second laser sources did not harm the surface passivation on $\mathrm{SiO}_{2}+\mathrm{a}-\mathrm{Si}: \mathrm{H}$ hardmask samples. It was possible to successfully pattern the base region by separate as well as overlapping pulses (with a maximum possible overlap of $10 \%$ between adjacent spots) without affecting the lifetime of minority carrier lifetime under the laser processed regions. From the range of fluences used in this work it was found that femto-second pulses $(515 \mathrm{~nm}$ and $1030 \mathrm{~nm}$ ) have a larger damage-free fluence window than nano-sec $(355 \mathrm{~nm})$ pulses. Thus, a simple and damage-free processing sequence for IBC-SHJ solar cells with optimal spot-to spot separation and fluence range was developed.

\section{Experimental}

In this study we investigated $\mathrm{SiO}_{2}$ and a stack of $\mathrm{SiO}_{2}+$ a-Si:H as the two hardmask structures for the emitter and base patterning sequence of IBC-SHJ cells as shown in Fig.1. The patterning of $\mathrm{SiO}_{2}+\mathrm{a}-\mathrm{Si}: \mathrm{H}$-mask samples is similar to the LEAP processing sequence [5]. The samples were prepared by depositing an a-Si:H(i) film on the front and back side of $\mathrm{c}-\mathrm{Si}(\mathrm{n})$ wafer as passivation layer and an a-Si:H(n) film on the back side as electron collecting layer. $\mathrm{An} \mathrm{SiO}_{2}$ film for the $\mathrm{SiO}_{2}$-mask samples, and an additional a-Si:H(i) films for the $\mathrm{SiO}_{2}+\mathrm{a}-\mathrm{Si}: \mathrm{H}$-mask samples were deposited on the back-side as shown in Fig.1a). Using laser ablation, openings were made in the $\mathrm{SiO}_{2}$ film and in the a$\mathrm{Si}: \mathrm{H}$ film for the respective samples (Fig1.b). In the $\mathrm{SiO}_{2}+\mathrm{a}-\mathrm{Si}$ :H-mask samples, the $\mathrm{SiO}_{2}$ film was etched in $\mathrm{HF}$. For both the samples, the underlying a-Si:H(i+n) layers were then etched in TMAH (Fig.1c). After a RCA cleaning step, the etched regions were re-passivated with an a$\mathrm{Si}: \mathrm{H}(\mathrm{i})$ film and an a-Si:H(p) film was deposited as the hole collecting layer (Fig.1d) on top. The impact on the passivation quality was studied after steps b) and d) in Fig.1 using photoluminescence imaging. In the last step of the LEAP sequence, the $\mathrm{SiO}_{2}$ layer can be etched in $\mathrm{HF}$ and the a-Si:H (i+p) above it can be lifted-off (Fig.1e) to form inter-digitated n-doped and p-doped regions[5]. This step was not tested in this study. To complete the fabrication of the solar cell a further metalization step is needed, which is not discussed here.

\subsection{Laser systems}

In this work, we have used $\mathrm{Yb}: \mathrm{KGW}$ based modelocked femto-second laser system from Light Conversion (type Pharos) and Nd:YVO 4 based Q-switched nanosecond laser system from Rofin (Powerline E series). Every laser beam had a Gaussian intensity distribution. Their specifications are as follows:

1) Laser system 1 (fs-VIS) : $\lambda=515 \mathrm{~nm} ; \tau_{\mathrm{p}}=200 \mathrm{fs} ; \omega_{\mathrm{o}}$ $=14.39 \mu \mathrm{m} @ 1 / \mathrm{e}^{2} ;$ Rep. frequency $=20 \mathrm{kHz}$

2) Laser system 2 (fs-IR) : $\lambda=1030 \mathrm{~nm} ; \tau_{\mathrm{p}}=200 \mathrm{fs} ; \omega_{\mathrm{o}}$ $=22.88 \mu \mathrm{m} @ 1 / \mathrm{e}^{2} ;$ Rep. frequency $=100 \mathrm{kHz}$

3) Laser system 3 (ns-UV): $\lambda=355 \mathrm{~nm} ; \tau_{\mathrm{p}}=6 \mathrm{~ns} ; \omega_{\mathrm{o}}=$ $12.56 \mu \mathrm{m} @ 1 / \mathrm{e}^{2} ;$ Rep. frequency $=15 \mathrm{kHz}$ 


\subsection{Sample preparation}

For the sample preparation, n-type (111) float-zone c-Si wafers were first cleaned using standard RCA cleaning, followed by passivation of both sides with $\sim 5 \mathrm{~nm}$ a-Si:H(i). Then $20 \mathrm{~nm}$ a-Si:H(n) as base contact layer and a $\sim 730 \mathrm{~nm} \mathrm{SiO} 2$ layer as the first hardmask were deposited on the entire wafer. An additional $\sim 75 \mathrm{~nm}$ a-Si:H was deposited on one half of the wafer to make the second hardmask structure. These two halves of the wafer with their respective hardmasks were used to make single-shot ablation experiments and to study microscopic properties, such as crater morphology and laser induced structural changes in the silicon wafer. The intrinsic a-Si:H(i) film was deposited using silane $\left(\mathrm{SiH}_{4}\right)$ and hydrogen $\left(\mathrm{H}_{2}\right)$, and the n- and pdoped a-Si:H films were deposited using additional phosphane $\left(\mathrm{PH}_{3}\right)$ and trimethylborane (TMB) gases in $\mathrm{AK} 1000$ inline RF- PECVD tool from Meyer Burger. The silicon dioxide film was deposited using silane $\left(\mathrm{SiH}_{4}\right)$ and nitrous oxide $\left(\mathrm{N}_{2} \mathrm{O}\right)$ in the Oxford PlasmaLab 100 PECVD tool. All the wet chemical steps were performed in Helmholtz Nano Facility (HNF)[11].

For photoluminescence imaging, a second set of samples was prepared as above. To avoid excessive etching through pinholes occurring in the a-Si:H(i)-mask we replaced this layer with an a-Si:H(p) film of similar thickness. On these samples, multiple square areas $\left(10 \times 10 \mathrm{~mm}^{2}\right)$ were ablated with separated and overlapping spots using all the three laser sources used in this study. The $\mathrm{SiO}_{2}+\mathrm{a}-\mathrm{Si}: \mathrm{H}-$ mask samples were then processed in $10 \% \mathrm{HF}$ for 5 minutes and both the samples were treated with 5\% TMAH at $25^{\circ} \mathrm{C}$ for 5 minutes to etch the underlying a-Si: $\mathrm{H}$ film. The samples were then RCA cleaned, re-passivated with $\sim 5 \mathrm{~nm}$ a-Si:H(i) and a $\sim 20 \mathrm{~nm}$ a-Si:H(p) was deposited on the back side as emitter layer. In another set of $\mathrm{SiO}_{2}$-mask samples, additional damage etching after the ablation step was performed in $25 \% \mathrm{TMAH}$ at $80^{\circ} \mathrm{C}$ for 30 minutes to remove any laser induced damage in the silicon wafer.

\subsection{Sample characterization}

\section{A. Microscopy measurements for morphology and threshold fluence}

The threshold fluences was evaluated by the method described by Liu[12]. The required measurement of crater diameter and morphology was performed using light and atomic force microscopes directly after laser ablation. Line scans of AFM images across the ablation crater were measured to study the evolution of crater profile with increasing fluence.

\section{B. Structural analysis by Raman spectroscopy}

Micro-Raman spectroscopy is a non-destructive tool to probe the crystalline state of the semiconductor material[13]. The Raman spectra arising from monocrystalline, polycrystalline and amorphous states of silicon are different and can be easily distinguished from each other using this technique. Any shift in the Raman peak, arising from the silicon wafer, indicates residual stress in the wafer due to laser processing. We measured the Raman spectra from the middle of the ablated spots, directly after laser ablation, using a $\mathrm{CW}$ laser with $\lambda=488 \mathrm{~nm}$ and spot diameter of $1 \mu \mathrm{m}$.

\section{Impact on minority carrier lifetime}

The minority carrier lifetime of a silicon wafer is related to the recombination activity on the surface of the wafer, considering a high enough bulk lifetime. Any damage to the intrinsic amorphous silicon (passivation layer) or the crystalline silicon surface underneath it during laser processing is reflected by a reduction in the local minority carrier lifetime in that region. Using a dynamic photoluminescence (PL) imaging method proposed by Ramspeck et al. [14] we were able to generate a spatially resolved minority lifetime mapping of our samples. This allowed us to study the effect of laser damage on the passivation quality with increasing fluence values and with decreasing spot-tospot distance. Spatially resolved PL measurements were carried out after laser ablation (Fig. 1b) and a-Si:H(i+p) deposition step (Fig.1d). All measurements were performed using an InGaAs camera in steady-state and transient mode.

\section{Results}

\subsection{Crater morphology}

The initial impact of single-shot laser pulses on both sample types - with $\mathrm{SiO}_{2}$-mask and with $\mathrm{SiO}_{2}+\mathrm{a}-\mathrm{Si}: \mathrm{H}(\mathrm{i})$ mask - was studied with AFM and light microscopy (LM). The inspection of surface topography and reflectivity changes using both these microscopes, gave complementary information about the effect of the different laser sources on the hardmasks, as the single pulse peak fluence was increased.

Fig. 2 shows the AFM images and Fig. 3 shows the corresponding $\mathrm{LM}$ images of the $\mathrm{SiO}_{2}$-mask samples treated with fs-VIS and ns-UV pulses with increasing fluence. As the quantitative results observed with fs-IR pulses were quite similar to those of fs-VIS pulses, they have not been shown here. At low pulse fluence, for both fs-VIS and nsUV pulses, a blister appears on the surface of the sample as seen in Fig.2a) and b). Here no direct ablation of $\mathrm{SiO}_{2}$ layer is observed from AFM images. The edge of this blister corresponds to the dark ring seen in the LM image of Fig.3a) and $b$ ). Outside this dark ring, another circular region with a color change with respect to the untreated region can be seen. The surface of this outer modified region is smooth and it is not detected in the AFM images. With the increase in pulse fluence, the size of the blister increases (Fig.2c and d), the corresponding dark ring along with the outer modification zone expands, and a third annulus appears in the center of the spot where the energy of the Gaussian pulse is highest (Fig.3c and d). Finally, above a certain critical fluence the blister delaminates and forms a crater (Fig.2e and f). The edge of this crater corresponds to the dark ring observed at lower fluences above. This crater edge, the inner annulus along with the outer modified region can also be observed in the microscope image of the delaminated crater (Fig.3c and d). 


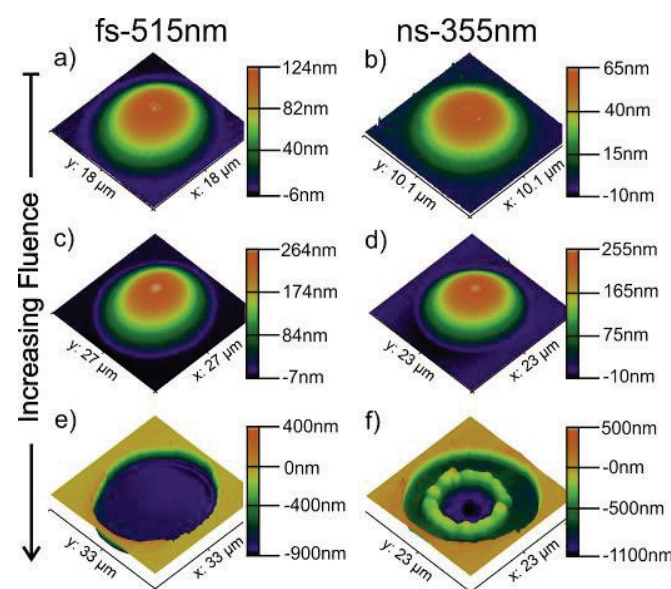

Fig.2 AFM images of $\mathrm{SiO}_{2}$-mask sample showing blister formation at low fluences and removal of $\mathrm{SiO}_{2}$ film at high fluence. With fs-pulses flat crater profile is obtained and with ns-pulses melting in the underlying wafer is observed.

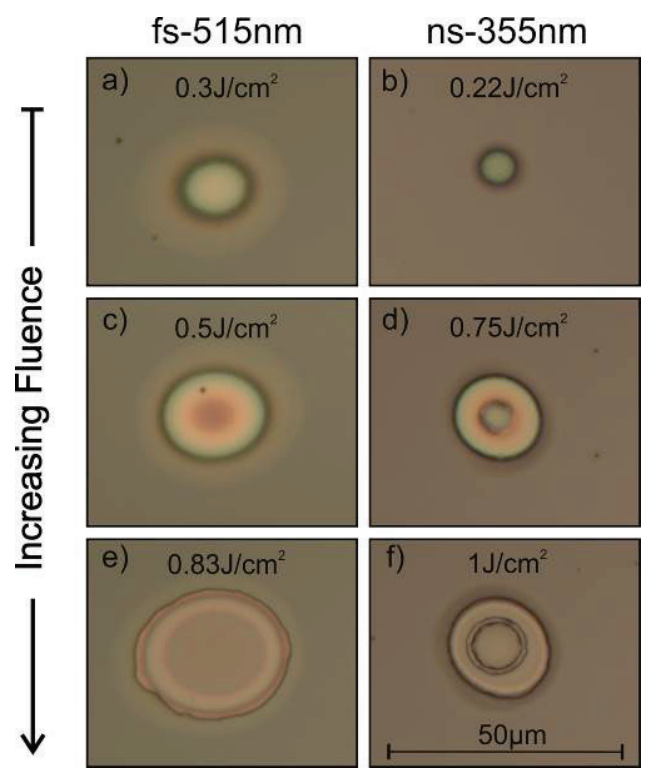

Fig.3 Light microscope images of $\mathrm{SiO}_{2}$-mask sample showing evolution of laser treated spots with increasing fluence. In e) and f) an outer modification zone with color change, the well-defined crater edge and an inner damage zones are clearly visible.

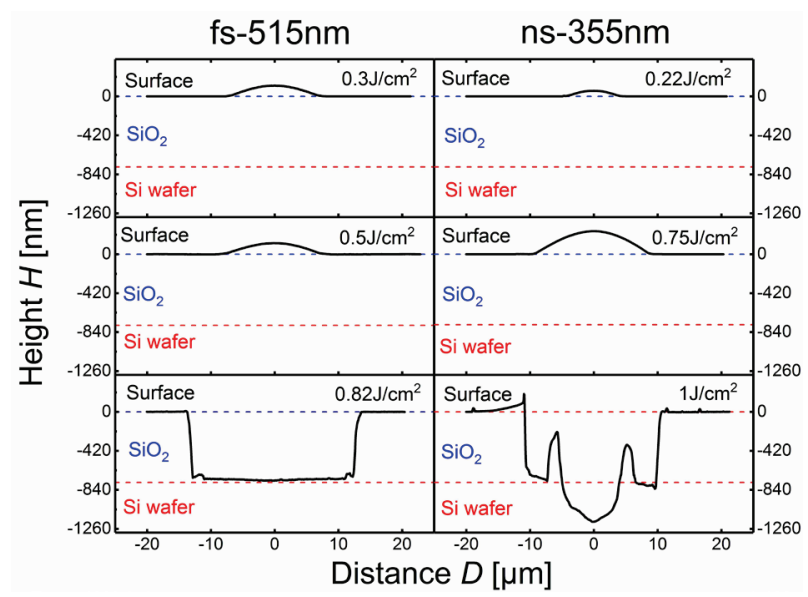

Fig.4 Evolution of laser spots with increasing fluence for the $\mathrm{SiO}_{2}$-mask sample.

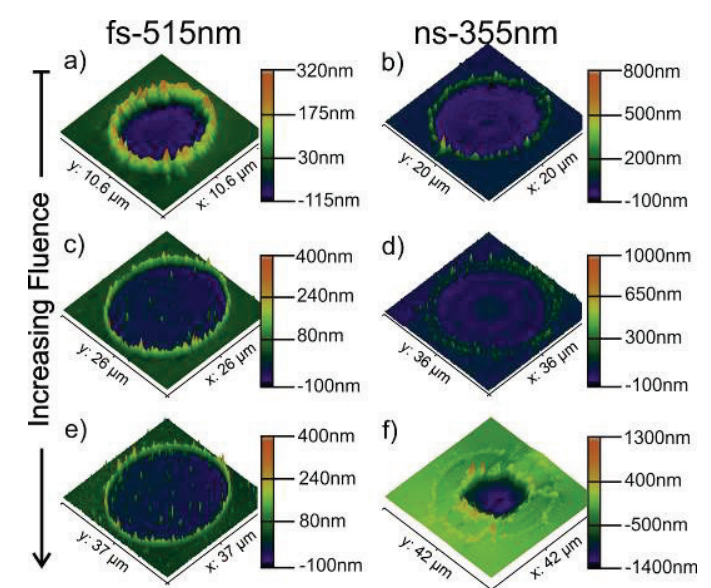

Fig.5 AFM images of $\mathrm{SiO}_{2}+\mathrm{a}-\mathrm{Si}: \mathrm{H}(\mathrm{i})$-mask sample showing ablation of a-Si:H(i)-mask with increasing fluence. With fs-pulses the crater depth saturates and with ns-pulses damage propagates to the silicon substrate with increasing fluences.

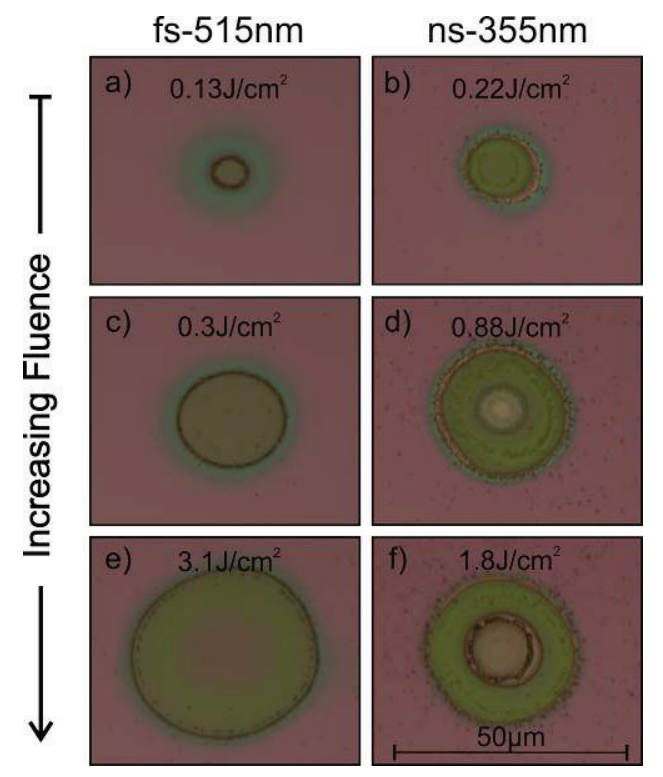

Fig.6 Light microscope images of $\mathrm{SiO}_{2}+\mathrm{a}-\mathrm{Si}: \mathrm{H}(\mathrm{i})$-mask sample showing evolution of laser treated spots with increasing fluence. In c) and d) an outer modification zone with color change and the well-defined crater edge are seen. For ns pulses an additional inner damage zone can be observed.

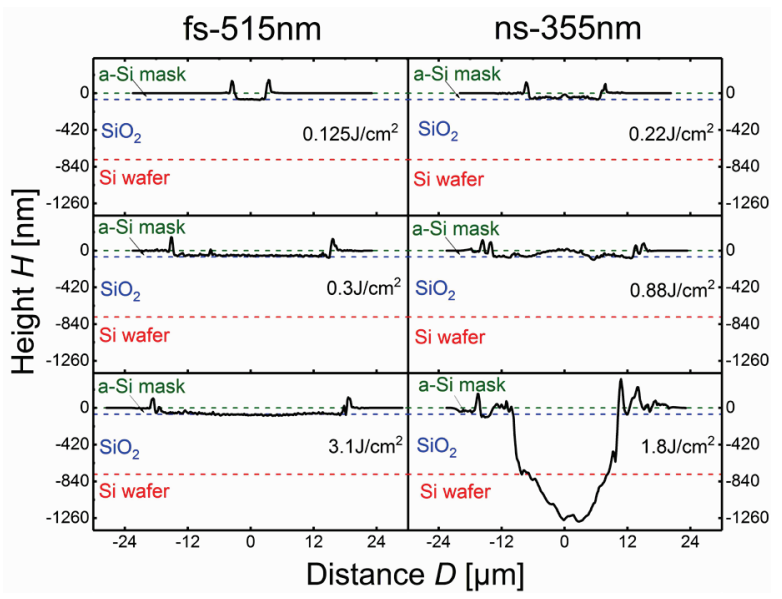

Fig.7 Evolution of laser spots with increasing fluence for the $\mathrm{SiO}_{2}+\mathrm{a}-\mathrm{Si}: \mathrm{H}(\mathrm{i})$-mask sample. 
The corresponding line scans across the laser treated spots are shown in Fig.4. The morphology of the crater is different for femto- and nano-second pulses. For femtosecond pulses the bottom of the crater has a flat profile and its depth of $\sim 760 \mathrm{~nm}$ corresponds to the thickness of the $\mathrm{SiO}_{2}$ mask and a-Si:H $(\mathrm{i}+\mathrm{n})$ layer beneath it. On increasing the fluence further, the surface of the crater becomes rougher but its depth does not increase significantly. For the nano-second pulses however, delamination is accompanied by molten debris of the underlying silicon wafer. This molten region in the middle corresponds to the inner circular region first observed in Fig.3c and d. The crater's depth increases with the fluence and is accompanied by greater volume of silicon being expulsed from the center.

For the $\mathrm{SiO}_{2}+\mathrm{a}-\mathrm{Si}: \mathrm{H}(\mathrm{i})$-mask sample the $\mathrm{LM}$ and AFM images of the laser processed spots with increasing fluence are shown in Fig.5 and 6 and the corresponding line scans across the AFM images is shown in Fig.7. As seen in Fig.5a) and b), in the low fluence regime, ablation of the top a-Si:H(i) mask takes place. The outer modification region and a ring of molten debris around the ablated crater are observed in the LM images (Fig.6 a and b). On increasing the pulse fluence, different ablation behavior is observed for femto- and nano-second pulses. For femtosecond pulses, the diameter of the crater increases but the depth remains constant even for very high peak fluences (Fig.5c and e). However, for nano-second pulses, as the fluence is increased a blister appears in the middle of the crater, corresponding to an inner ring seen in the LM image (Fig.6d). The blister can be better observed in the line scan given in Fig.7. The evolution of this blister is similar to that observed earlier with the $\mathrm{SiO}_{2}$-mask sample. With increasing fluence the blister expands, followed by delamination of underlying $\mathrm{SiO}_{2}$ layer and a high damage to the silicon wafer underneath it (Fig.5f).

\subsection{Threshold fluence measurements}

From these observations we defined three different laser treated zones during the processing of $\mathrm{SiO}_{2}$-mask sample for threshold fluence calculations. The outer circular region, with a reflectivity change but without any topographical modification (Fig. 3a and b), was defined as the 1) modification zone. The dark ring around the blister, which eventually forms the edge of delaminated crater (Fig.3e and f), corresponds to the 2) a-Si:H/c-Si ablation zone. Finally, in the inner ring observed at medium and high fluences (Fig. $3 \mathrm{c}-\mathrm{f}$ ) we found structural changes in the underlying silicon substrate using Raman spectroscopy (as shown in section 3.3). We defined this region as the 3) damage zone.

Similarly, for a-Si:H+SiO${ }_{2}$-mask sample we defined the outer ring (Fig.6a and b) as the 1) modification zone and the ring surrounding the ablation crater as the 2) a-Si:H(i)mask ablation zone. For ns-pulse irradiation, the inner ring seen in Fig.6d) and f) can be associated with the damage to the underlying a-Si:H/c-Si. This zone was defined as 3) damage zone.

The individual diameters of each of the abovementioned zones were measured with increasing fluence value and the squared diameter values were plotted against the logarithm of pulse fluence. Threshold fluence of each mechanism was then obtained by fitting the data with a linear fit and extrapolating the fitted lines to the $\mathrm{X}$-axis, where the diameter of each zone tends to zero. These results for $\mathrm{SiO}_{2}$-mask and $\mathrm{SiO}_{2}+\mathrm{a}-\mathrm{Si}: \mathrm{H}$ mask sample are presented in Fig.8 and Fig.9 respectively and summarized in Table 1 and 2 .

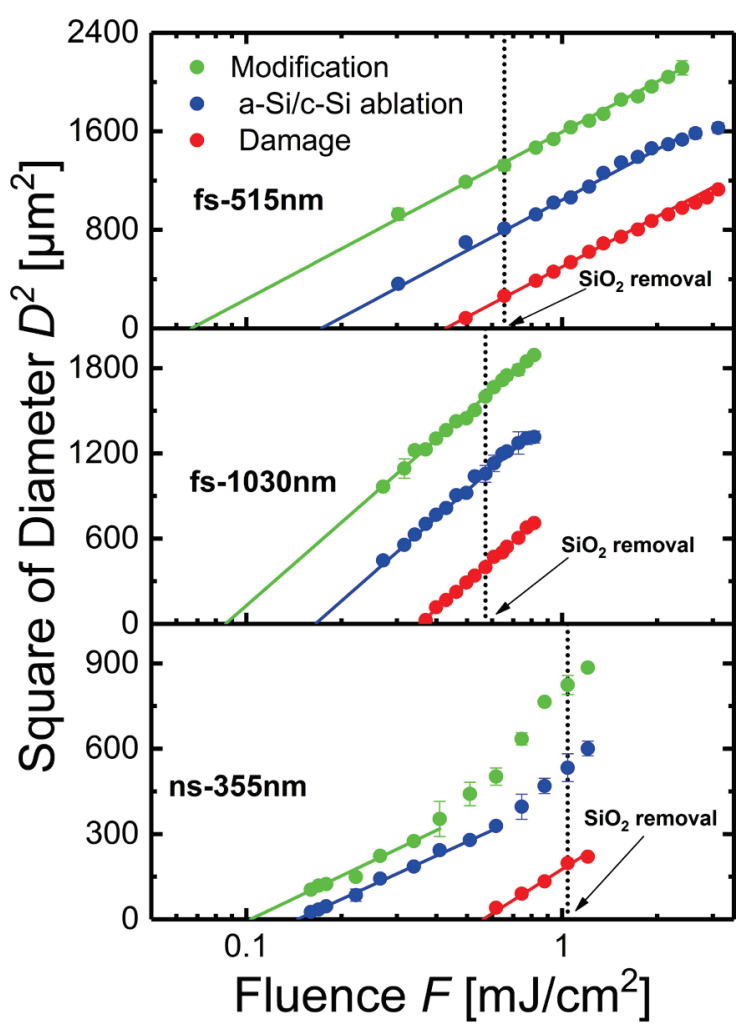

Fig.8 Threshold fluence determination of surface modification, a$\mathrm{Si}: \mathrm{H} / \mathrm{c}-\mathrm{Si}$ ablation and damage zones for $\mathrm{SiO}_{2}$-mask sample. Dotted line indicates the fluence beyond which in-direct ablation of $\mathrm{SiO}_{2}$ film takes place. For all the laser sources, damage is induced in the substrate (red curve) before the indirect-ablation of $\mathrm{SiO}_{2}$ film takes place.

Table 1 Threshold fluences for modification, ablation and damage processes on $\mathrm{SiO}_{2}$-mask sample obtained by irradiation with femto-second $(\lambda=515 \mathrm{~nm}, 1030 \mathrm{~nm})$ and nanosecond $(\lambda=355 \mathrm{~nm})$ laser pulses.

$\mathrm{SiO}_{2}$-mask

$\begin{array}{ccc}\text { Modification } & \begin{array}{c}\text { Ablation } F_{\text {th }} \\ F_{\text {th }}\left(\mathrm{J} / \mathrm{cm}^{2}\right)\end{array} & \begin{array}{c}\text { Damage } F_{\text {th }} \\ \left(\mathrm{J} / \mathrm{cm}^{2}\right)\end{array} \\ \end{array}$

$\begin{array}{cccc}\text { fs- } & 0.066 \pm 0.015 & 0.172 \pm 0.01 & 0.426 \pm 0.004\end{array}$

fs-

$\begin{array}{llll}1030 n m & 0.085 \pm 0.004 & 0.165 \pm 0.013 & 0.353 \pm 0.009\end{array}$

ns-

$0.102 \pm 0.008 \quad 0.144 \pm 0.003$

$0.561 \pm 0.022$ 


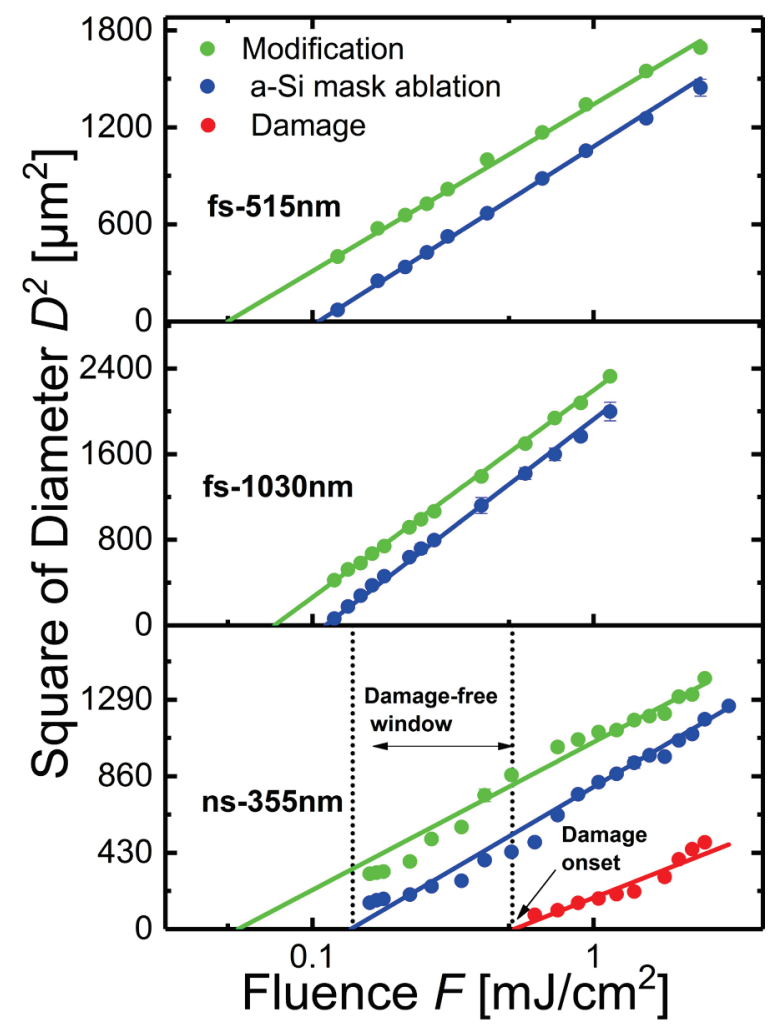

Fig.9 Threshold fluence determination of surface modification, a$\mathrm{Si}: \mathrm{H}$ mask ablation and damage zones for $\mathrm{SiO}_{2}+\mathrm{a}-\mathrm{Si}: \mathrm{H}(\mathrm{i})$-mask sample. Dotted lines indicate the damage-free ablation window obtained for nano-second pulses.

Table 2 Threshold fluences for modification, ablation and damage processes on $\mathrm{SiO}_{2}+\mathrm{a}-\mathrm{Si}: \mathrm{H}$ mask sample obtained by irradiation with femto-second $(\lambda=515 \mathrm{~nm}, 1030 \mathrm{~nm})$ and nano-second $(\lambda=355 \mathrm{~nm})$ laser pulses.

\begin{tabular}{cccc}
\hline \multicolumn{4}{c}{ SiO $_{2}+\mathbf{a - S i}:$ H-mask } \\
\hline & $\begin{array}{c}\text { Modification } \\
F_{\text {th }}\left(\mathrm{J} / \mathrm{cm}^{2}\right)\end{array}$ & $\begin{array}{c}\text { Ablation } F_{\text {th }} \\
\left(\mathrm{J} / \mathrm{cm}^{2}\right)\end{array}$ & $\begin{array}{c}\text { Damage } F_{\text {th }} \\
\left(\mathrm{J} / \mathrm{cm}^{2}\right)\end{array}$ \\
\hline $\begin{array}{c}\text { fs- } \\
\mathbf{5 1 5 n m}\end{array}$ & $0.049 \pm 0.007$ & $0.104 \pm 0.004$ & - \\
$\mathbf{f s -}$ & $0.073 \pm 0.003$ & $0.111 \pm 0.001$ & - \\
$\mathbf{1 0 3 0 n m}$ & & & \\
$\mathbf{n s -}$ & $0.080 \pm 0.013$ & $0.138 \pm 0.008$ & $0.510 \pm 0.028$ \\
\hline $\mathbf{3 5 5 n m}$ & & & \\
\hline
\end{tabular}

For the $\mathrm{SiO}_{2}$-mask samples, the experimentally determined fluence for the complete removal of $\mathrm{SiO}_{2}$ is indicated in Fig. 8 with a dotted black line. As can be seen from the figure, the delamination of $\mathrm{SiO}_{2}$ film occurs at a higher fluence than the onset of damage fluence (red line) for each of the laser sources used in this work. For a-Si:H-mask sample no visual damage was observed within the craters formed by femto-second pulses at high fluence values. This indicates that there is a large damage-free processing win- dow with femto-second pulses. On the other hand, for nano-second pulses, we notice that there is only a small damage-free processing window in the initial fluence regime which is below the onset of damage fluence of a$\mathrm{Si}: \mathrm{H} / \mathrm{c}-\mathrm{Si}$ interface. This window is marked in Fig.9.

\subsection{Structural analysis using Raman spectroscopy}

Micro-Raman spectra were obtained from the middle of the ablation craters and from reference unprocessed regions for both the sample types and for each of the laser sources used in the study. These results are displayed in Fig.10 and 11.

The reference spectrum from $\mathrm{SiO}_{2}$-mask sample (Fig.10) can be best fitted by two peaks - one well defined sharp peak centered at $520 \mathrm{~cm}^{-1}$ and another weaker and broader peak around $470 \mathrm{~cm}^{-1}$. The intensity of the weaker peak has been multiplied thirty times for better visibility. The first peak is well-known from literature to be associated with the optical mode (LO+TO) of unstressed monocrystalline Si (SI-I) [15] and the secondary peak can be associated with that of amorphous silicon. Thus, in the reference position the contribution in Raman spectra was from the silicon substrate and amorphous silicon $(\mathrm{i}+\mathrm{n})$ layers below the $\mathrm{SiO}_{2}$-mask. For each of the ablated crater formed by the different laser sources we measured a broader and asymmetric TO-peak at $520 \mathrm{~cm}^{-1}$ with a shoulder at lower wavenumbers. This peak was de-convoluted into the central $520 \mathrm{~cm}^{-1}$ peak and a secondary peak centered between $516 \mathrm{~cm}^{-1}$ and $518 \mathrm{~cm}^{-1}$, as shown in Fig.10. This shift in peak position at lower wavenumbers can be attributed to the formation of polycrystalline silicon during melting, and subsequent recrystallization of the silicon wafer with a slight tensile stress caused by laser damage. We did not observe any Raman signal around $470 \mathrm{~cm}^{-1}$ in the craters. This indicates the removal of amorphous silicon $(i+n)$ layers during laser ablation.

As shown in Fig.11, for $\mathrm{SiO}_{2}+\mathrm{a}-\mathrm{Si}: \mathrm{H}$-mask sample the reference spectrum from the unprocessed region was heavily attenuated by the top $75 \mathrm{~nm}$ a-Si:H mask. The signal strength was weak but a broad peak around $470 \mathrm{~cm}^{-1}$ emanating from the top a-Si:H layer and an attenuated signal at $520 \mathrm{~cm}^{-1}$ from the unstressed c-Si substrate can be clearly observed. In the Raman spectra from the ablated spots (created by different laser sources) a symmetrical central c-Si peak at $520 \mathrm{~cm}^{-1}$ and a very faint contribution from amorphous silicon around $470 \mathrm{~cm}^{-1}$ can be resolved. The fitted spectra at $470 \mathrm{~cm}^{-1}$ have been multiplied twenty times to make them visible. This amorphous silicon signal is similar to the reference spectra of $\mathrm{SiO}_{2}$ sample without the top a$\mathrm{Si}: \mathrm{H}$ mask and it could be attributed to a-Si:H (i+n) passivation and base contact layer below the $\mathrm{SiO}_{2}$ layer. 


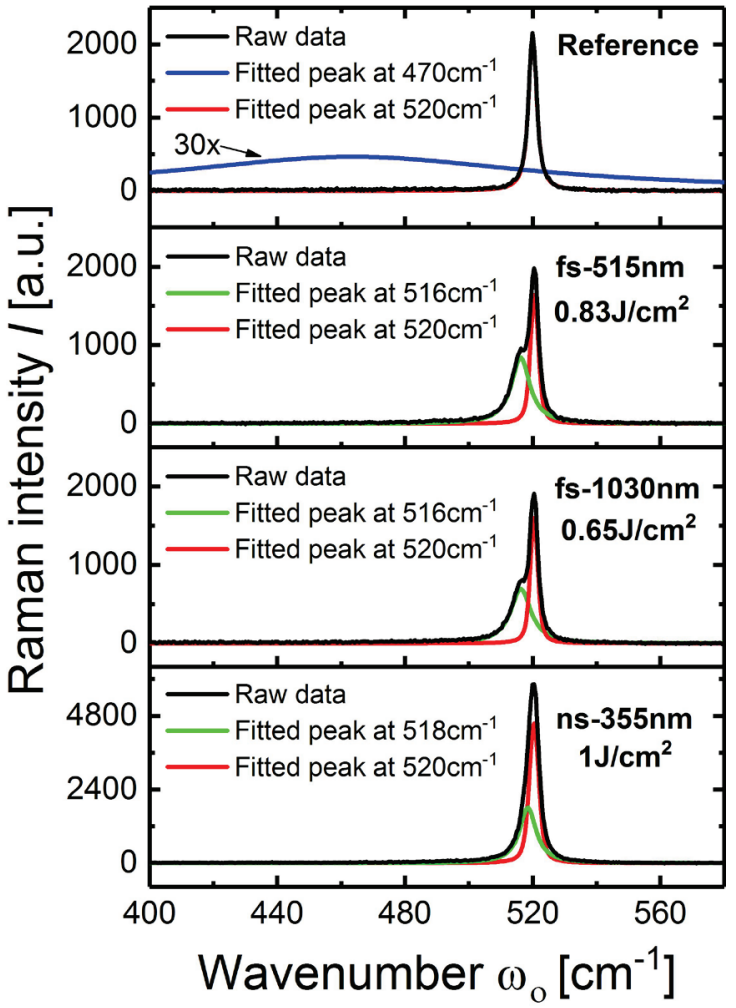

Fig.10 Raman spectra of $\mathrm{SiO}_{2}$-mask sample, obtained from reference region, and from the center of ablation craters. The Raman signal coming from the c-Si substrate $\left(520 \mathrm{~cm}^{-1}\right)$ shows a clear modification after laser ablation as compared to reference region indicating damage due to laser processing.

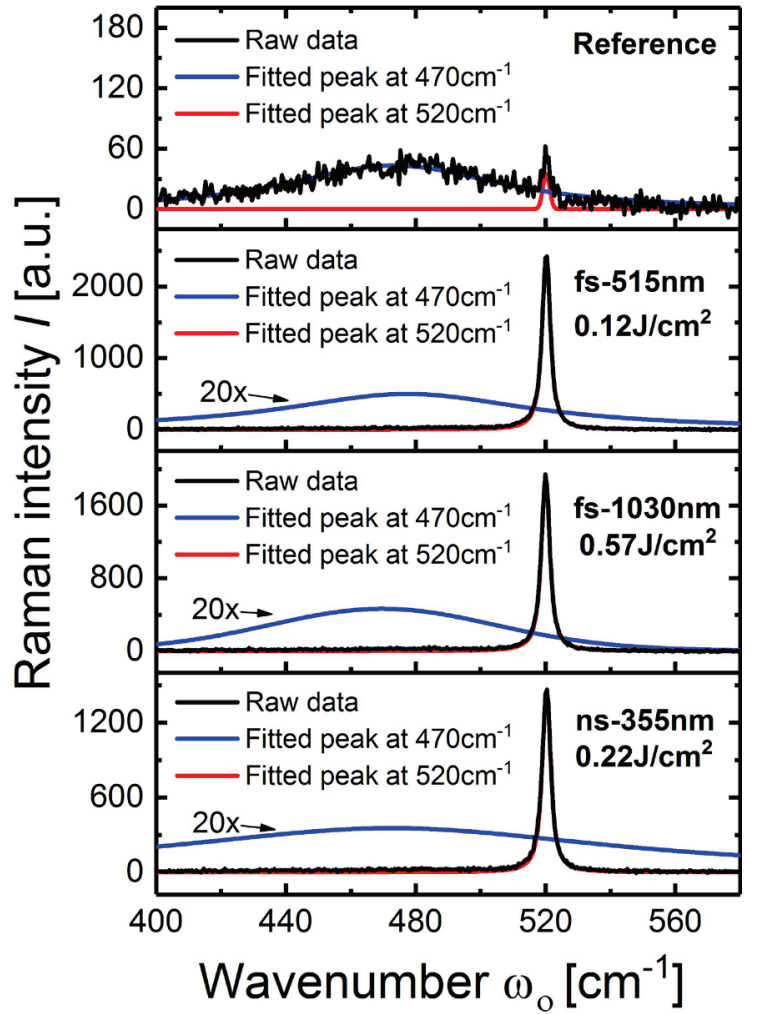

Fig.11 Raman spectra of $\mathrm{SiO}_{2}+\mathrm{a}-\mathrm{Si}: \mathrm{H}(\mathrm{i})$-mask sample, obtained from reference region and from the center of ablation craters. In the reference region, the signal corresponding to $\mathrm{c}-\mathrm{Si}$ peak (at $\left.520 \mathrm{~cm}^{-1}\right)$ is attenuated by the a-Si:H(i) mask. After ablation of the mask, the c-Si signal strength increases. No modification in this cSi Raman peak (emanating from the wafer) is observed after ablation indicating an undamaged substrate after laser processing.

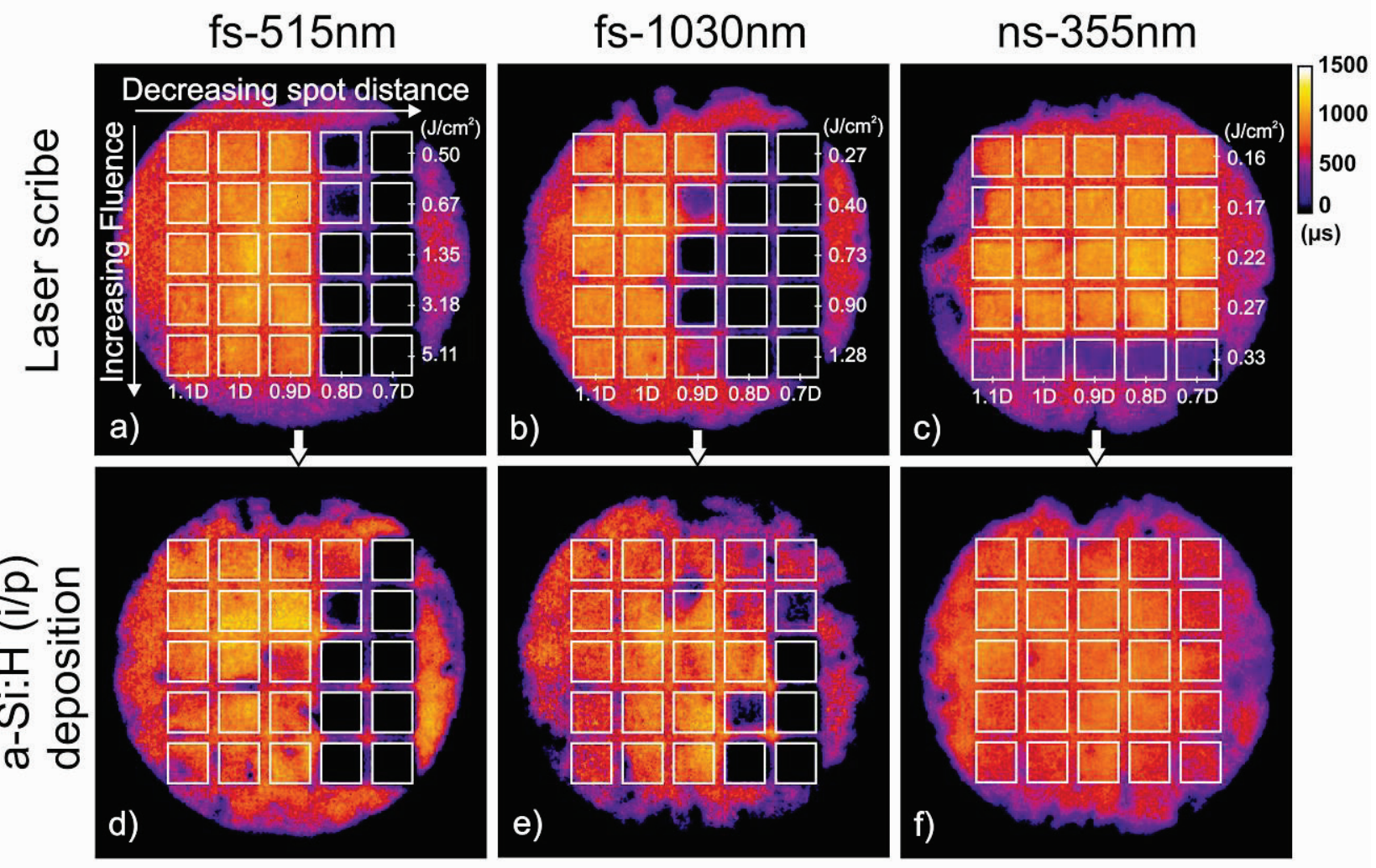

Fig.12 Minority carrier lifetime mapping of $\mathrm{SiO}_{2}+\mathrm{a}-\mathrm{Si}: \mathrm{H}(\mathrm{i})$-mask sample after laser scribing step (a-c) and after a-Si:H(i+p) deposition step (d-f) using fs-515nm, $1030 \mathrm{~nm}$ and ns-355nm laser pulses. As exemplified in a) in each sample, multiple square regions were processed with decreasing spot distance (left to right) and increasing laser fluence (top to bottom). The distances between the spots were reduced in proportion to the individual crater diameter $-\mathrm{D}$ for each fluence value. 


\subsection{Effect on minority carrier lifetime}

To test the damage to the c-Si/a-Si:H interface, $\mathrm{SiO}_{2}-$ mask samples were treated by single-shot laser pulses with peak fluence greater than the experimentally determined threshold delamination fluence required to ablate the top $\mathrm{SiO}_{2}$ mask. The photoluminescence-lifetime mapping result showed severe reduction in the minority carrier lifetimes for all the three laser sources (not shown here), thus indicating a high amount of damage induced by the laser treatment. The minority carrier lifetime remained low after etching the sample in TMAH, RCA cleaning and subsequent re-passivation (Fig.1- steps $\mathrm{c}$ and d). This lifetime remained low even for the $\mathrm{SiO}_{2}$-samples in which an additional damage-etch was performed in TMAH at high concentration and temperature.

In case of the $\mathrm{SiO}_{2}+\mathrm{a}-\mathrm{Si}: \mathrm{H}$ mask sample it was observed from preliminary experiments that the ablation of the top a-Si:H mask with single laser spots did not reduce the minority carrier lifetime. As the spots were brought closer in the ablation step (Fig.1-b), complete etching of the underlying $\mathrm{SiO}_{2}$ mask and the lift-off of unprocessed top a-Si:H mask in the laser processed region took place during the etching step (Fig.1-c). This happened due to the lateral etching of $\mathrm{SiO}_{2}$ from under the un-ablated regions in-between the ablated spots. However, when the spots were brought too close to each other, damage in the underlying c-Si/a-Si substrate occurred in the overlapping zone of the spots. Therefore, an optimal spot-to-spot distance corresponding to the minimum distance between two adjacent spots, which does not induce any damage in the underlying layers while also allowing fast $\mathrm{SiO}_{2}$ etching below the a-Si:H mask, is desired for a simple processing sequence.

Based on these findings we compared the laser sources to determine a damage-free processing window of fluence and an optimal spot-to-spot distance between adjacent laser spots. To do so, we varied the fluence of the femto-second lasers within a large range of values and the fluence of the nano-second laser just beyond the damage threshold window, which was determined by the threshold fluence measurement in section 3.2 (see Table 2). To find out the optimal spot-to-spot distance, separated laser spots were brought closer to each other in steps, and subsequently overlapped in both $\mathrm{X}$ and $\mathrm{Y}$ directions. Their impact on the minority carrier lifetime was observed by using the photoluminescence-lifetime mapping.

The minority carrier lifetime images taken after laser scribe step (Fig.1-b) and after a-Si:H(i+p) deposition step (Fig.1-d) using all the three laser sources used in this work, are shown in Fig.12.The laser processed areas are marked with square rectangles. In the laser scribe step (Fig.12 a-c), the fluence was increased from top to bottom, and from left to right the distance between the individual spots was reduced in proportion to the individual spot diameters - D. This is exemplified in Fig.12a). The first two columns of each laser scribed sample correspond to separated spots (1.1D and 1D), and the last three columns correspond to overlapping spots $(0.9 \mathrm{D}, 0.8 \mathrm{D}$ and $0.7 \mathrm{D})$.

For femto-second lasers we notice in Fig.12a) and b) (column 1 and 2) that the minority carrier lifetime is not affected by single spot ablation within the range of fluence values used in our work $\left(0.5-5.1 \mathrm{~J} / \mathrm{cm}^{2}\right.$ for fs-515nm and
$0.27-1.3 \mathrm{~J} / \mathrm{cm}^{2}$ for fs-1030nm). From left to right, as the spots are brought close to each other, we notice a severe reduction in minority carrier lifetime in the laser processed regions (column 4 and 5). After the etching of underlying $\mathrm{SiO}_{2}$ layer and a-Si:H (i+p) deposition, we notice from Fig.12d) and e) that the lifetimes in some of the borderline damaged regions (column 4) could be revived, especially in the case of fs-1030nm pulses. This points to the fact that damage occurred within the a-Si:H(i+n) layer on top of the silicon wafer surface in these regions. In the regions with high pulse-to-pulse overlap the reduction in the minority carrier lifetime could not be improved after the a-Si:H(i+p) deposition step. In these regions, we observed some wavy patterns in the overlapping zones under the light microscope which resemble the well-known laser induced periodic surface structures(LIPSS)[16].

For the ns-UV laser the fluence was varied from 0.16 to $0.33 \mathrm{~J} / \mathrm{cm}^{2}$. The upper value corresponds to the edge of the damage-free window determined in section 3.2. Indeed, we notice a reduction in the minority carrier lifetime for the highest fluence value (Fig.12c) with separated and overlapping pulses. However, the reduction in the minority carrier lifetime is not as severe as that observed in the case of femto-second pulses with overlapping pulses. From Fig.10e) we see that this damage is completely recovered after the a-Si:H (i+p) deposition step. This indicates that the damage is confined within the a-Si:H(i+n) layer for the highest fluence value, and that there is room to further increase the fluence in order to ascertain the permanent damage fluence limit with nano-second pulses. From left to right, as the spot distance is reduced we do not see any reduction in minority carrier lifetime. On a closer microscope inspection, we noticed that due to pulse-to pulse energy variation and lower beam quality for the nano-second laser (giving rise to elliptical shape of the spots), the pulses did not sufficiently overlap within the ablated zone. Nevertheless, during the etching process all the silicon dioxide film was etched and the non-ablated a-Si:H(i) mask between the ablation crater was lifted-off within a short HF dip.

Hence we were able to successfully use laser ablation process to remove a-Si:H(i+n) layer and deposit a$\mathrm{Si}: \mathrm{H}(\mathrm{i}+\mathrm{p})$ using both the expensive femto-second laser as well as cost-effective nano-second laser without harming the minority carrier lifetimes of the sample.

\section{Discussion}

The ablation mechanism of $\mathrm{SiO}_{2}$ films on bare silicon has been studied by other groups in the literature [[9], [17] [21]] and a similar blister formation and subsequent delamination of the top $\mathrm{SiO}_{2}$ film has been reported by them. Their findings can be used to explain the ablation behavior of our $\mathrm{SiO}_{2}$-mask sample. In the ablation of $\mathrm{SiO}_{2}$-mask sample discussed in section 3.1 no evidence of direct ablation of $\mathrm{SiO}_{2}$ layer is observed. It can be concluded that during laser irradiation, the pulse energy is mostly transmitted by the $\mathrm{SiO}_{2}$ film and is absorbed within the underlying c$\mathrm{Si} / \mathrm{a}-\mathrm{Si}: \mathrm{H}$ interface. At peak fluences greater than the melting threshold of the interface, local melting and expansion of a small volume of the material takes places below the $\mathrm{SiO}_{2}$ film. If this fluence is below the breaking threshold of $\mathrm{SiO}_{2}$ film, incomplete lift-off the $\mathrm{SiO}_{2}$ film results in a blister-like feature. When the fluence exceeds the breaking 
threshold of $\mathrm{SiO}_{2}$ film, the energy deposited in the underlying a-Si:H/c-Si stack creates enough stress (due to high vapor pressure) to completely lift-off the top $\mathrm{SiO}_{2}$ film via indirect ablation. In the ablated craters we found no evidence of amorphous silicon films as compared to reference spectra taken from $\mathrm{SiO}_{2}$-mask samples. For femto-second ablation, the depth of the crater corresponds well with the thicknesses of the $\mathrm{SiO}_{2}$ layer and underlying a-Si:H layers. For ns-laser, clear ablation of silicon wafer was evident in the AFM measurements. This indicates that the delamination of $\mathrm{SiO}_{2}$ takes place when sufficient energy is absorbed, not only in the a-Si:H $(\mathrm{i}+\mathrm{n})$ layer but also in the silicon wafer itself, to create enough stress for the lift-off of $\mathrm{SiO}_{2}$ film.

In the case of femto-second ablation, the crater bottom had a flat profile and its depth did not increase significantly with increasing the fluence. One possible explanation for this observation could be related to the fact that the absorption of femto-second pulses in silicon is a non-linear process which causes an instantaneous excitation of a large density of electrons [22]. This produces electron-hole plasma with quasi-metallic absorption properties that limits the penetration depth of the pulse energy within a small volume on the silicon surface. The decay of the electronhole plasma happens at the characteristic timescale of electron-phonon interaction of around 1ps [23].Therefore, for $\sim 200 \mathrm{fs}$ long pulses a high carrier density can be expected. For nano-second pulses no such electron-hole plasma is produced. As a result, we obtain an increasingly large amount of ablation from the middle of the crater as the peak fluence is increased.

The Raman measurement of $\mathrm{SiO}_{2}$-mask sample (Fig.10) from the middle of ablated crater showed an unstressed c-Si peak at $520 \mathrm{~cm}^{-1}$ and a secondary peak at lower wavenumbers. In the literature this secondary peak is associated with poly-silicon phase. The explanation of ablation behavior of $\mathrm{SiO}_{2}$-mask sample is consistent with this observation. The ablation behavior can thus be explained as follows: to achieve the delamination of $\mathrm{SiO}_{2}$ film, local melting of silicon surface occurs. Due to the presence of large heat gradient outside the ablated region rapid resolidification takes place and the melted silicon solidifies into polycrystalline phase.

From the ablation threshold measurements (Fig.8) it was experimentally determined that the delamination of the $\mathrm{SiO}_{2}$ film occurs at fluences greater than the damage threshold fluence of underlying surface. This was true for all the lasers used in this work. From the Raman spectra we confirmed structural modification of the silicon surface by the laser process. This explains the severe degradation of minority carrier lifetimes of $\mathrm{SiO}_{2}$-maks sample after laser ablation. The etching of the $\mathrm{n}(111)$ silicon wafer in $25 \% \mathrm{TMAH}$ at $80^{\circ} \mathrm{C}$ was not sufficient enough to remove all the damaged areas, hence the minority charge carrier lifetimes could not be improved during re-passivation.

In $\mathrm{SiO}_{2}+\mathrm{a}-\mathrm{Si}: \mathrm{H}$-mask sample, ablation of the top a-Si:H layer by femto-second laser showed similar saturation of crater depths with increasing fluences as in the case of $\mathrm{SiO}_{2}$-mask samples (Fig.7). As discussed above, formation of electron-hole plasma caused by multi-photon absorption in a-Si:H layer could be the explanation for this observation. For nano-second pulses on the other hand, no such electron-hole plasma is formed and once the complete a$\mathrm{Si}: \mathrm{H}$ layer is ablated, the surplus energy is transmitted through the $\mathrm{SiO}_{2}$ film, leading to damage in the passivation layer. For higher fluences, similar blister formation and delamination takes place, as seen in $\mathrm{SiO}_{2}$-mask sample.

For femto-second pulses, we only observed the ablation of the a-Si:H mask, but no topographical modification in between the craters for the range of fluence values used in this work. Furthermore, the Raman spectra from the ablated spots showed an undamaged c-Si peak at $520 \mathrm{~cm}^{-1}$ originating from the underlying silicon, and a much smaller yet detectable a-Si:H peak around $480 \mathrm{~cm}^{-1}$ originating from the underlying a-Si:H $(\mathrm{i}+\mathrm{n})$ layers. Hence, it can be concluded that single-shot ablation of $\mathrm{SiO}_{2}+\mathrm{a}-\mathrm{Si}: \mathrm{H}(\mathrm{i})$-mask sample does not induce damage in the c-Si/a-Si:H interface. This result was further confirmed by the PL-lifetime analysis in which no reduction in minority carrier lifetime was observed by single-shot femto-second pulses. The absorption of higher pulse energies by the electron-hole plasma allowed a large damage-free processing window.

For nano-second $(355 \mathrm{~nm})$ pulses we observed an undamaged c-Si peak only within a small process window using the Raman measurements. The minority carrier lifetime was unaffected within this fluence window, after laser processing. Beyond this fluence window, degradation in the minority carrier lifetime was observed for single nanosecond $(355 \mathrm{~nm})$ pulses.

All the laser spots could be overlapped until $10 \%$ of their individual diameters without inducing any damage to the minority carrier lifetime of the substrate. At this optimal distance, the complete lift-off of remaining unabated a-Si:H mask (via lateral etching of underlying $\mathrm{SiO}_{2}$ ) took place within $2 \mathrm{~min}$ in $10 \%$ HF dip. For higher overlaps (fs-pulses) and higher fluence (ns-pulses), damage was induced in the substrate. In some of the borderline damaged regions, the passivation quality was recovered after a-Si:H(i+n) etching and $\mathrm{a}-\mathrm{Si}: \mathrm{H}(\mathrm{i}+\mathrm{p})$ deposition step. This indicates that the damage in these regions was confined only in the a$\mathrm{Si}: \mathrm{H}(\mathrm{i}+\mathrm{n})$ layer.

\section{Conclusion}

We tested two hard mask structures for implementation in the back-side patterning of IBC-SHJ solar cells. Crater morphology was studied by means of atomic force and light microscopy. The ablation behavior of both the hardmask was studied with increasing laser fluences. Successful damage-free ablation was demonstrated with a combination of $\mathrm{SiO}_{2}$ and a-Si:H hard mask as evident from high minority carrier lifetimes, measured from the laser processed areas using photoluminescence imaging method. This result was also supported by Raman measurements. A processing window of fluence and spot-to-spot distance was obtained for each of the laser pulses. IBC-SHJ solar cell fabrication using the selected a-Si:H hard-mask, and the use of spots with $10 \%$ overlap is planned for future work.

\section{Acknowledgement}

This project has received funding from European Union's Horizon2020 program (under Grant Agreement no. 727523) and the Helmholtz Energy Materials Foundry (HEMF). 
The authors would like to thank Yael Augarten, Andrew Wrigley and Vito Huhn for the photoluminescence imaging set-up and various fruitful discussions around imaging method; Markus Hülsberg for helping us with the Raman measurements and lastly Gunnar Schöpe and Andreas Bauer for their support with handling the laser set-up.

\section{References}

[1] S. De Wolf, A. Descoeudres, Z. C. Holman, and C. Ballif: Green, 2, (2012) 7.

[2] J. Nakamura, N. Asano, T. Hieda, C. Okamoto, H. Katayama, and K. Nakamura: IEEE J. Photovoltaics, 4, (2014) 1491.

[3] M. A. Green, Y. Hishikawa, Dean H. Levi, E. D. Dunlop, and J. Hohl-Ebinger: Prog. Photovolt. Res. Appl., 26, (2018) 3.

[4] S. Harrison, O. Nos, G. D’Alonzo, C. Denis, A. Coll, and D. Munoz: Energy Procedia, 92, (2016) 730.

[5] S. Ring,S. Kirner,C. Schultz, P. Sonntag, B. Stannowski, and L. Korte, R. Schlatmann: IEEE J. Photovoltaics, 6, (2016) 894.

[6] M. Xu,T. Bearda,M. Filipic,H. S. Radhakrishnan, M. Debucquoy, I. Gordon, J. Szlufcik, and J. Poortmans: Proc. 44th IEEE PVSC, (2017)

[7] J. Krüger and W. Kautek: Laser Phys., 9, (1999) 30.

[8] "Key criteria for choosing a laser manufacturer can be established by a single attribute,", Available online at : https://ktu.edu/uploads/files/MMI/industriallasers20170 506-dl.pdf\#page=1\&zoom=auto,-124,783.

[9] T. Rublack, M. Schade, M. Muchow, H. S. Leipner, and G. Seifert: J. Appl. Phys., 112, (2012) 023521.

[10]P. Engelhart, S. Hermann, T. Neubert, H. Plagwitz, R. Grischke, R. Meyer, U. Klug, A. Schoonderbeek, U. Stute, and R. Brendel: Prog. Photovoltaics Res. Appl.,15, (2007) 521.

[11]W. Albrecht, J. Moers, and B. Hermanns: J. large-scale Res. Facil.,3, (2017) A112.

[12]M. J. Liu: Opt. Lett., 7, (1982) 196.

[13]F.H.Pollak: "Analytical Raman Spectroscopy" ed. by J. G. Grasselli,B. J. Bulkin, (Wiley, New York, 1991) p.137.

[14]K. Ramspeck, S. Reissenweber, J. Schmidt, K. Bothe, and R. Brendel: Appl. Phys. Lett., 93, (2008) 102104.

[15]M. S. Amer, L. Dosser, S. LeClair, and J. F. Maguire: Appl. Surf. Sci., 187, (2002) 291.

[16]B. Tan and K. Venkatakrishnan: J. Micromechanics Microengineering, 16, (2006) 1080.

[17]T. Rublack, M. Muchow, S. Hartnauer, and G. Seifert: Energy Procedia, 8, (2011) 467.

[18]T. Rublack, S. Hartnauer, M. Mergner, M. Muchow, M. Schade, H.S.Leipner, and G.Seifert: Proc. SPIE, 8247, (2012) 82470Z.

[19]S. Rapp, M. Domke, M. Schmidt, and H. P. Huber: Phys. Procedia, 41, (2013) 734.

[20]S. Hermann, T. Dezhdar, N. P. Harder, R. Brandel, and M. Seibt: J. Appl, Phys., 108, (2010) 114514.

[21]S. Hermann, N. P. Harder, R. Brendel, D. Herzog, and H. Haferkamp: Appl. Phys. A, 99, (2009) 151.

[22]N. Medvedev and B. Rethfeld: J. Appl. Phys., 108, (2010) 103112.

[23]J. R. Goldman and J. A. Prybyla: Phys. Rev. Lett., 72, (1994) 1364. 